\title{
Softwares educacionais podem ser instrumentos psicológicos
}

\author{
Softwares educacionais: instrumentos psicológicos
}

\author{
Cristiano Mauro Assis Gomes
}

\begin{abstract}
Resumo
( texto defende o pressuposto de que os softwares educacionais podem ser modelados de modo a se tornarem instrumentos potenciais de alteração do fluxo de desenvolvimento cognitivo dos estudantes. Em conjunto a esse pressuposto há o argumento de que os softwares educacionais, com o objetivo de alterar o desenvolvimento dos estudantes devem ser instrumentos psicológicos. Três "grandes ondas" descrevem e demarcam diferentes relações entre a educação e os softwares educativos. Para análise dessas relações, artigos de revistas internacionais em ensino de ciências foram coletados, na medida em que tradicionalmente o ensino de ciências tem apresentado importantes inovações nessa área. $O$ texto conclui seus argumentos salientando a função dos softwares educacionais na construção do pensamento dos alunos. Concomitantemente destaca o papel dos softwares no desenvolvimento cognitivo e profissional dos professores.

Palavras-chave: desenvolvimento cognitivo; aprendizagem, medida.
\end{abstract}

\section{Educational software can be psychological tools}

\begin{abstract}
The text defends that educational software can be fashioned in order to become potential instruments of alteration on the students' cognitive development course. The educational software has the objective of modifying the development of the students and must be psychological tools. Three "great waves" describe different relationships between the education and educational software. To analyze these relationships, they had collected articles from the international journals in sciences education for the reason that traditionally the education of sciences has presented important innovations in this area. The text concludes its arguments pointing out the function of educational software in the construction of the students' thought. At the same time it detaches the role of software in the teacher's cognitive and professional development.

Keywords: cognitive development; learning; measurement.
\end{abstract}

\section{Softwares educacionales pueden ser instrumentos psicológicos}

\section{Resumen}

El texto defiende el presupuesto de que los softwares educacionales pueden ser modelados de forma a tornarse instrumentos potenciales de alteración del flujo de desarrollo cognitivo de los estudiantes. Junto a ese presupuesto está el argumento de que los softwares educacionales, con el objetivo de alterar el desarrollo de los estudiantes, tienen que ser instrumentos psicológicos. Son descriptas tres "grandes olas" que marcan diferentes relaciones entre la educación y los softwares educativos. Para el análisis de esas relaciones fueron cosechados artículos de revistas internacionales en enseñanza de ciencias, una vez que tradicionalmente la enseñanza de ciencias ha presentado importantes innovaciones para el área. El texto concluye sus argumentos destacando la función de los softwares educacionales en la construcción del pensamiento de los alumnos. Junto a eso también se destaca el papel de los softwares en el desarrollo cognitivo y profesional de los profesores.

Palabras clave: desarrollo cognitivo; aprendizaje, medida. 


\section{Educação e informática: uma relação de múltiplas facetas}

Desde o início da década de 1970, o computador tem sido utilizado por vários países como um instrumento para o desenvolvimento e a melhoria dos processos de ensino-aprendizagem. Diversos softwares foram produzidos no intuito de estabelecer tecnologias de ensino-aprendizagem e essa produção passou a ser denominada na literatura internacional de CbEs Computer-based Education (Cotton, 1991). Analisando esse cenário, pode-se dizer que o campo tem passado por transformações significativas ao longo do tempo, no que tange às relações constituídas entre a educação e a informática, assim como à construção e o uso dos softwares educacionais (Berger, Lu, Belzer \& Voss, 1994). Duas extensas meta-análises realizadas por Berger e colaboradores (1994) e por De Jong e Van Joolingen (1998) apresentam evidências a respeito da ocorrência dessas transformações.

Enquanto a meta-análise de Berger e colaboradores (1994) enfocou as principais pesquisas internacionais que tinham como objeto de investigação a confecção e utilização de softwares educacionais nos anos de 1970 e 1980, no campo do ensino de ciências, a metaanálise de De Jong e Van Joolingen (1998) analisou essa mesma temática entre os anos de 1980 e 1990. As evidências de Berger e colaboradores (1994) mostram que nos anos de 1970 havia uma primazia de softwares com princípios comportamentais e positivistas e que essa predominância foi alterada substancialmente nos anos de 1980 para a produção e o uso de softwares educacionais voltados para a construção do conhecimento e a alteração dos esquemas mentais. Segundo os autores, essa mudança profunda foi gerada porque uma nova perspectiva educacional foi inaugurada, subsidiando uma articulação entre a educação e a informática bem diferente da anterior. De Jong e Van Joolingen (1998), por sua vez, encontraram transformações também muito relevantes entre os anos de 1980 e 1990 e apontaram para uma nova relação entre a educação e a informática que passou a determinar a construção e o uso de softwares como meios simbólicos, voltados a propiciar aos estudantes uma assimilação mais efetiva das estruturas cognitivas sociais encontradas nos conteúdos escolares e uma interação sujeito-objeto de maior qualidade. Semelhante ao período anterior, uma nova perspectiva educacional subsidiou essa mudança.

Neste texto, três grandes tendências que articulam a relação entre o uso dos softwares educacionais, a informática e o campo da educação serão discutidas. Discuti-las será útil porque a última delas abre possibilidades reais e efetivas para o pressuposto defendido neste texto: o de que os softwares educacionais podem ser instrumentos psicológicos. Ao mesmo tempo, a apresentação dessas diferentes perspectivas situará um conjunto variado de possibilidades sobre a interação entre a educação e a informática.

Descrevendo essas tendências, a primeira "grande onda" surgiu no início da década de 1970. Nesse período, a informática e o computador tinham como função educacional servir como instrumento precioso na transmissão clara e precisa das informações presentes nos conteúdos curriculares escolares. Além da transmissão, os softwares educacionais deveriam oferecer ambientes com tarefas de fixação e reforço da aprendizagem para alterar o comportamento dos estudantes (Whithaus, 2004). Essa tendência foi muito forte a ponto de influenciar a quase totalidade das pesquisas internacionais a respeito do papel educacional dos softwares (Berger e cols., 1994). A maior parte dos estudos nos anos de 1970 centrou seu foco nos efeitos ocasionados pelos CbEs sobre a aprendizagem dos estudantes, através da análise da quantidade da informação adquirida versus o tempo de aquisição da informação.

A partir dessa primeira conexão entre a informática e a educação nasceram os softwares de Instrução Assistida por Computador, os CAls (Computer-Assisted Instruction). Nos anos de 1970, os CAls se estruturaram na concepção de que uma informação precisa e sem ruídos garantiria uma aprendizagem melhor. As unidades de cada conteúdo eram expostas ao estudante em pequenas porções de informação, seguidas por um conjunto de exercícios ou atividades capazes de modelar os comportamentos emitidos pelos alunos, em função do princípio da aquisição seqüencial e gradativa do conteúdo assim como da repetição da transmissão. Novas unidades eram transmitidas quando o aluno demonstrava um conjun- 
to mínimo de respostas adequadas de fixação das informações e execução dos problemas (Cotton, 1991; Karlgren, 2006).

Apesar do vigor apresentado no início dos anos de 1970, a primeira "onda" cedeu lugar a uma segunda "grande onda" que demarcou uma nova forma de vinculação entre a informática e a educação. Essa nova tendência foi marcada pelo princípio educacional construtivista da aprendizagem por descoberta (Sjøberg, 2007; Vasconcelos, Praia \& Almeida, 2003) e que tinha como um de seus focos principais a interação sujeito-objeto e a elaboração de ambientes capazes de oferecer o maior grau de liberdade ao sujeito nessa interação. No campo do ensino de ciências, a maior parte das pesquisas nos anos de 1980 sobre o uso do computador passou a avaliar o papel dos softwares nas interações espontâneas dos estudantes com os objetos de conhecimento presentes nos ambientes virtuais dos softwares. Esses estudos passaram a analisar como as interações espontâneas e os conflitos cognitivos propiciados por elas ou induzidos pelo professor poderiam alterar as concepções prévias dos alunos e gerar novo conhecimento (De Jong \& Van Joolingen, 1998).

Um dos postulados educacionais mais fortes da segunda onda é encontrado na concepção de que a construção do conhecimento poderia ser garantida a partir de ambientes virtuais ricos capazes de permitir ao sujeito tanto uma interação livre com os objetos de conhecimento, como a possibilidade de vivenciar conflitos cognitivos que desestabilizassem os seus esquemas de conhecimento prévios de modo a gerar novos conhecimentos. O modelo construtivista de ensino que subsidiou a segunda onda defendia a concepção de que a interação sujeito-objeto e a formação de conflitos cognitivos seriam capazes de promover a construção do conhecimento e uma aprendizagem efetiva por parte dos estudantes. Schur (1997) argumenta que esse modelo surgiu inicialmente para a comunidade internacional através de um artigo seminal de Driver e Easley (1978). A partir desse período, o campo do ensino de ciências, influenciando a educação escolar de uma maneira mais geral, voltou-se para observar e alterar os esquemas mentais de conhecimentos específicos dos alunos, elabo- rados a partir da interação sujeito-objeto. As estratégias pedagógicas dessa época centravam-se no objetivo de alterar os esquemas de conhecimento dos estudantes através da geração de conflitos cognitivos. Acreditava-se que os conflitos cognitivos teriam o poder de desestabilizar os esquemas mentais prévios dos estudantes e, concomitantemente, propiciar espaço para que novos esquemas pudessem ser construídos. Nesse contexto, em essência o professor deveria ser um provocador de conflitos cognitivos e, os softwares, por conseqüência, deveriam ajudar nessa missão.

Experimentações Simuladas por Computador (CSEs - Computer-Simulated Experimentation) e Laboratórios baseados em Microcomputador (MbLs Microcomputer-based Laboratories) foram produtos dessa proposta educacional, indicando respectivamente ambientes que simulam experimentações e favorecem a aquisição de conhecimentos declarativos e procedimentais de pesquisa e experimentação, assim como ambientes que simulam materiais, ferramentas e procedimentos presentes em um ambiente de laboratório (Kubicek, 2005), ambos favorecendo a interação sujeito-objeto.

Apesar do caráter inovador trazido pela segunda "grande onda", ela passou a ser alvo de críticas sistematizadas e de amplo alcance a partir da década de 1990, justamente por valorizar excessivamente os processos de interação individual do estudante com os objetos de conhecimento e apostar demasiadamente na capacidade do conflito cognitivo. Boa parte das críticas à segunda onda baseou-se no argumento de que a aprendizagem por descoberta, levada aos seus extremos, ocasionou uma série de empecilhos e lacunas na condução pedagógica do processo de aprendizagem. A meta-análise de De Jong e Van Joolingen (1998), já abordada neste texto, mostrou evidências de que boa parte das interações sujeito-objeto alcançadas em vários softwares educacionais da segunda onda não possuía uma qualidade minimamente aceitável. Segundo os autores, o problema encontrado não se referia à qualidade dos softwares, em termos de sua adequação à proposta educacional vigente da época. Ao contrário, eles atendiam aos princípios estabelecidos. Uma das conclusões elaboradas pelo 
estudo afirmava que os ambientes virtuais que se concentravam exclusivamente em oferecer possibilidades ricas de interação sujeito-objeto não eram capazes de gerar uma aprendizagem de qualidade nos alunos. Outra conclusão afirmava que os conflitos cognitivos não eram facilmente gerados nesse tipo de ambiente. Por sua vez, quando eram gerados, normalmente eles apenas desestabilizavam os esquemas mentais dos estudantes sem propiciar a construção de conhecimentos epistemologicamente superiores aos esquemas prévios.

Para sustentar suas conclusões, os autores apresentaram vários problemas encontrados pelos alunos em suas interações com ambientes virtuais de aprendizagem de caráter construtivista que apresentavam um forte potencial para gerar interações ricas entre $\circ$ sujeito e o objeto e, por conseqüência, conflitos cognitivos. O primeiro problema citado pelos autores envolvia o desenvolvimento do pensamento científico e a forte limitação dos estudantes em gerar hipóteses. Analisando extensos relatos de pesquisas, os autores verificaram que os estudantes que interagiam com ambientes de experimentação sem nenhum cerceamento, tutoria ou dicas, mostravam dificuldades em gerar hipóteses. Boa parte dos estudantes apresentava consideráveis dificuldades em estabelecer relações entre as variáveis dos ambientes virtuais assim como em relacionar a teoria com os dados observados em ambientes simulados. Em algumas situações, os estudantes construíam hipóteses rígidas e não as alteravam mesmo a partir de informações contrárias fornecidas pelas experimentações realizadas nos softwares. Os alunos também apresentavam problemas em construir desenhos experimentais.

Eles elaboravam experimentos considerando apenas variáveis que poderiam comprovar as suas hipóteses iniciais, não planejavam e articulavam os elementos importantes para uma exploração do ambiente simulado, de forma que o desenho experimental não possuía relação com as variáveis contidas nas hipóteses, o que invalidava a experimentação. Outro aspecto que mostrava severas lacunas na interação entre os alunos e os objetos de conhecimento envolvia a interpretação de dados. Os estudantes apresentavam uma interpretação errônea dos dados provenientes dos experimentos realizados em ambientes simulados com uma dificuldade pronunciada na área da interpretação de gráficos. No entanto, o problema mais grave identificado foi a dificuldade dos estudantes em regular a sua própria aprendizagem. Em geral, os estudantes com baixo desempenho escolar apresentavam importantes limitações no monitoramento de suas próprias ações, indicando uma dificuldade na maneira de interagir com os objetos de conhecimento dos ambientes virtuais. Na medida em que esses ambientes ou o professor não atuavam na forma como o aluno poderia elaborar estratégias para monitorar a sua própria aprendizagem, boa parte dos estudantes permanecia no mesmo lugar, ou seja, não alterava seus processos metacognitivos de interação com os objetos de conhecimento.

Um dos motivos centrais que ocupou a crítica à segunda onda envolveu, pois, a desconfiança de que $\circ$ processo educativo deveria se centrar na interação entre o sujeito e o objeto e no conflito cognitivo como propulsores fundamentais da construção do conhecimento. Pesquisadores como Schur (1997), Solomon (1994), Feuerstein, Rand e Rynders (1988), entre vários outros, passaram a argumentar claramente que ambientes extremamente ricos necessitavam ser desvendados e desbravados, pois careciam de uma interação mais sofisticada a qual a maior parte dos alunos não estava preparada, se deixada por conta própria, ou se provocada exclusivamente através de conflitos cognitivos gerados pelo ambiente virtual ou pelo professor. Essa crítica implicava que a interação sujeito-objeto deveria ser subsidiada ou complementada por outras estratégias pedagógicas e não apenas pelo princípio do ambiente rico e do conflito cognitivo. Mediações entre o sujeito e o objeto precisariam ser empregadas para que o estudante pudesse alterar de fato seus esquemas mentais prévios e viesse a construir novos conhecimentos em níveis epistemologicamente mais sofisticados.

A crítica à segunda onda gerou a terceira grande onda, acrescentando-se um novo vínculo entre a informática e a educação. Segundo De Jong e Van Joolingen (1998), esse novo vínculo passou a determinar nos anos de 1990 a agenda principal das pesquisas internacionais do ensino de ciências sobre o papel da 
informática e dos softwares, envolvendo tanto a análise dos ambientes virtuais, em termos de sua riqueza para a interação sujeito-objeto (preocupação da segunda onda), como os processos mediacionais necessários para que a interação sujeito-objeto pudesse ser ampliada de forma a gerar conhecimento de qualidade (preocupação acrescentada pela terceira onda).

A próxima seção tratará especificamente das características principais da terceira onda, assim como também abordará o argumento principal defendido neste texto: o de que softwares educacionais podem ser instrumentos psicológicos.

\section{Softwares Educacionais podem ser Instru- mentos Psicológicos}

A terceira onda pretende que o computador e os softwares educacionais sejam capazes de provocar mudanças na qualidade da interação sujeito-objeto. A perspectiva educacional que subsidia essa nova onda parte do princípio de que as interações sujeito-objeto podem ser alteradas substancialmente quando é alterada concomitantemente a capacidade de pensar do aluno, seja através de intervenções pedagógicas em suas formas de raciocinar, representar, elaborar estratégias, etc (Kozma, 1991). Há um conjunto de postulados comuns na terceira onda que podem ser descritos da seguinte maneira (Salomon, Perkins \& Globerson, 1991):

I. Os ambientes virtuais têm o potencial de permitir ao estudante pensar em um nível superior ao que ele é capaz de forma espontânea, alterando progressivamente suas estruturas cognitivas. 2. As habilidades cognitivas e as estratégias de pensamento fomentadas em ambientes virtuais digitais podem ser generalizadas para situações e eventos gerais, o que sugere ser o computador um potente instrumento para o desenvolvimento da capacidade de aprendizagem.

3. Os ambientes virtuais podem desenvolver nos estudantes uma prontidão para a análise do seu próprio pensamento, impulsionando a metacognição e a construção de uma aprendizagem profunda.

Esses pontos reúnem vários pesquisadores (Bliss \& Ogborn, 1992; Courtois, 1992; Hron, 1992;
Jonassen, 1996; Mandl \& Hron, 1992; Nirmalakhandan, 2007; Tisseau, 1992) em um universo comum: o de que os softwares podem ser modelados para atuarem como ferramentas na reestruturação do pensamento de maneira que novas formas de interação entre o sujeito e o objeto sejam possíveis. Corroborando essa tese, Mayes (1992) e Jonassen (1992), integrantes dessa proposta, sustentam que qualquer software pode tornar-se um instrumento cognitivo, se puder:

I. Ter um grau de extensão frente aos processos cognitivos humanos, independentemente se foi inicialmente criado para isso ou não.

2. Fomentar ações cognitivas gerais.

3. Assistir o processo de pensamento do aluno.

4. Agir como instrumento que facilite e engaje $\circ$ estudante em um processamento significativo da informação através da construção do conhecimento.

A preocupação com a re-estruturação do pensamento e a noção de que os softwares podem ser meios simbólicos capazes de modificar as formas de interação dos sujeitos com os objetos de conhecimento abre margem para que se possa relacionar teoricamente essa posição junto ao conceito de instrumento psicológico de Vygotsky (Vygotsky; 1978, 1986, 1987; Wertsch, 1985; Zinchenko, 1985). Conforme destaca Kozulin (1999), o pensamento vygotskiano foi fortemente influenciado pelo pensamento de Janet de que as funções mentais superiores ocorrem primeiro no plano intersubjetivo das interações sociais para depois serem interiorizadas e construídas internamente no plano intra-subjetivo. As análises de Kozulin (1999) indicam que a influência do postulado de Janet ajudou Vygotsky a elaborar novos argumentos a respeito do papel dos meios simbólicos culturais na formação das estruturas cognitivas individuais (Kozulin, 1999). Um desses argumentos enfatizava que as estruturas cognitivas sociais deveriam ser assimiladas pelo sujeito para que novas estruturas cognitivas individuais pudessem ser geradas e 0 sujeito pudesse atingir novos níveis em seu desenvolvimento mental. Kozulin (2000) demonstra que o conceito vygotskiano de instrumento psicológico envolve o problema da assimilação das estruturas soci- 
ais de pensamento por parte do sujeito. Em termos processuais, um instrumento psicológico é gerado quando o sujeito é capaz de assimilar os processos fundamentais presentes em um meio simbólico da cultura, elaborando internamente uma nova ferramenta mental capaz de lhe oferecer novos níveis de pensamento e análise da realidade (Kozulin, 2000).

Um argumento forte da terceira onda defende a interação entre a educação e a informática como uma oportunidade para a geração de novas formas de pensamento através da construção mediada do conhecimento. Em suma, nessa visão o pensamento deve ser mais deliberadamente construído, impulsionado e alterado por intervenções educacionais bem direcionadas e focadas. Essa posição educacional possui fortes relações com os conceitos de meios simbólicos e instrumentos psicológicos de Vygotsky, na medida em que há uma crença comum nessas posições de que determinados agentes sociais externos podem alterar profundamente a capacidade de pensar do ser humana, de suas estruturas internas, sua autonomia cognitiva e, por conseqüência, a sua capacidade para interagir com os objetos de conhecimento.

Enquanto a segunda seção deste texto tratou da terceira onda e do princípio de que softwares educacionais podem ser instrumentos psicológicos, a terceira seção expande $\circ$ argumento principal deste texto e acrescenta que softwares educacionais podem ser instrumentos psicológicos tanto para alunos como para professores.

\section{Softwares Educacionais podem ser Instru- mentos Psicológicos tanto para Alunos como para Professores}

Para Vygotsky (1987), a capacidade de pensar de uma pessoa é produto do modo como ela adquire os meios simbólicos de sua cultura. $O$ pensamento é alterado em nível estrutural através da interiorização de novos meios simbólicos. Na medida em que os meios simbólicos tornam-se interiorizados e dominados por um indivíduo, o pensamento se altera, novos componentes cognitivos são gerados, assim como novas formas de representação e de interação com os objetos de conhecimento. É nesse sentido que surge um novo instrumento psicológico. Apesar do alto grau de possibilidades oferecidas pelos meios simbólicos, na realidade o processo de incorporação desses meios não é um fenômeno simples (Kozulin, 2000). Roth (1995) encontrou evidências de que os alunos possuem considerável dificuldade em assimilar os meios simbólicos presentes nos conteúdos curriculares escolares, o que dificulta a produção de significados e o desenvolvimento de novas estruturas cognitivas. Essas evidências são corroboradas por vários pesquisadores de modo a indicar um fenômeno generalizado (Andaloro, Bellomonte \& SperandeoMineo, 1997; Beichner, 1994; Thornton \& Sokoloff, 1990).

Ao que sugerem certas pesquisas (Kozulin, 2000), a condução do processo de assimilação das estruturas sociais de pensamento presentes nos conteúdos curriculares escolares e a transformação dos meios simbólicos em instrumentos psicológicos é um processo trabalhoso que demanda muito engajamento e know-how profissional por parte do professor, envolve materiais didáticos específicos para essa finalidade e requer métodos pedagógicos sofisticados. Levando em consideração essas dificuldades, certos softwares educativos podem ser entendidos como oportunidades profissionais. Explicando o que isso quer dizer, argumento que alguns softwares educacionais podem ajudar o professor a desenvolver a sua capacidade de ser um mediador mais incisivo na capacidade de pensar e na constituição efetiva de instrumentos psicológicos em seus alunos. Ampliando o argumento, certos softwares educacionais podem atuar como ferramentas que se acoplam de forma imbricada na ação docente de mediação junto ao aluno, abrindo potenciais para uma alteração significativa na atuação profissional docente. Hatley (2007), por exemplo, mostra que os melhores resultados pedagógicos têm sido provocados quando professor e computador formam uma interação rica e de auxílio, a qual eu preferia chamar e entender como uma interação imbricada. Mas o que seria essa interação imbricada? É possível que o estudo de Doerr (1997) ajude na explicação desse conceito.

Doerr (1997) mostra que certos ambientes simulados imprimem a oportunidade de aulas diferenciadas, capazes de provocar processos cognitivos de 
alto nível. Essas aulas demandam aos alunos, entre outras coisas:

I. Elaborar e testar hipóteses alternativas, pondo em prática seus modelos.

2. Operar sobre vários níveis de variáveis, analisando seus efeitos dentro de um sistema qualquer.

3. Construir uma variedade de representações sobre quaisquer fenômenos estudados, utilizando diversas modalidades de linguagem e níveis de abstração.

4. Modelar e experimentar eventos demasiadamente lentos ou rápidos, muito caros, complexos ou perigosos, mudando escalas, valores etc (Schroeder \& Moore, 1993).

$\mathrm{O}$ aspecto mais interessante do estudo de Doerr (1997) não se refere exclusivamente ao papel dos softwares junto ao desenvolvimento cognitivo dos alunos. Seu estudo possibilita a especulação teórica de que os softwares educacionais podem penetrar intensamente na maneira do professor dar aula, mobilizar suas estratégias e ações docentes de modo a criar o que estou chamando de interação imbricada, em que o software passa a não ser apenas uma extensão da ação do professor para provocar a aprendizagem do aluno, mas modifica a sua própria ação. Esse contexto, evidentemente, é diferente do uso do computador em separado, pois o professor dá a sua aula e usa "em anexo" um software, seja para transmitir alguma informação, expor um conteúdo etc.

Juntando os estudos de Doerr (1997), o argumento de que os softwares podem ser instrumentos para $\circ$ desenvolvimento profissional do professor e o argumento ampliado de que é possível uma interação imbricada entre o professor e certos softwares, é possível construir um novo argumento, o de que os softwares podem ser instrumentos cognitivos para 0 próprio professor. No entanto, essa situação é condicionada a situações em que os softwares interferem diretamente na maneira do professor de atuar e trabalhar, alterando o seu fluxo de produção e criação. Por exemplo, teoricamente, um professor pode desenvolver a sua capacidade de ensinar aos alunos como construir várias formas de representar e organizar mentalmente os conceitos de um campo de conhecimento através de ambientes virtuais elaborados especificamente para gerar diferentes modalidades e níveis de representação da realidade.

Em uma interação imbricada com o software, o professor pode elaborar aulas em que os alunos aprendam a identificar e construir diferentes representações sobre um mesmo fenômeno. $O$ mais significativo disso, e aí reside o argumento teórico defendido, é que nessa interação imbricada em que o professor ensina os seus alunos a representar, ele pode desenvolver tanto a sua competência profissional de alterar a capacidade de pensar dos estudantes como a sua própria capacidade de representar fenômenos. Ao trabalhar intimamente com meios simbólicos nos softwares elaborados para provocar o pensamento do aluno não é pouco provável que o próprio professor tenha seu pensamento provocado.

Jonassen (1996) apresenta os softwares como instrumentos da mente (MindTools), termo cunhado por ele para dizer sobre o potencial do computador e dos softwares em alterar estruturas e padrões cognitivos das pessoas. Aproveitando o termo de Jonassen (1996), argumento de usar softwares de forma imbricada e como instrumentos da mente, o professor tem a chance de desenvolver as suas estruturas cognitivas. Nesse contexto, não somente os estudantes têm as suas mentes provocadas e transformadas. Isso é o que se espera quando se argumenta que alguns softwares educacionais podem ser instrumentos psicológicos tanto para os alunos como para os professores. Ao usar uma ferramenta cognitiva como meio de ensino que visa à transformação cognitiva do aluno, o professor tem grande chance como mediador humano de ser impulsionado cognitivamente (Kozulin, 2000). Ao buscar transformar, o agente da transformação pode ser transformado.

\section{Conclusões}

A cada nova ferramenta de pensamento, novas formas de interpretar e relacionar a realidade são disponibilizadas, abrindo potenciais latentes de novos fluxos de desenvolvimento e de aprendizagem. Os meios simbólicos presentes nos softwares educacio- 
nais são elementos potenciais de mudança dos indivíduos. No entanto, nada disso é possível sem a atuação do agente humano, ou seja, do professor como mediador entre os meios simbólicos e o indivíduo. $\mathrm{Na}$ medida em que os meios simbólicos são modulados, agenciados e reconstruídos nas interações sociais, determinadas interações sociais se fazem mais significativas e centrais na aquisição dos meios simbólicos pelos estudantes (Kozulin, 2000). Pode-se afirmar que nem toda interação social é capaz de provocar efeitos cognitivos significativos. Não basta colocar os alunos para interagirem consigo próprios e com os ambientes dos softwares para que a aprendizagem e a construção do conhecimento desponte. Nesse sentido, o papel do professor é central. No entanto, determinados softwares disponibilizam elementos que confluem para uma potencialização na alteração do desenvolvimento cognitivo dos estudantes e na ampliação de sua capacidade de aprendizagem.

Ao mesmo tempo em que o professor é agente e mediador fundamental da construção do estudante, as estruturas cognitivas do professor podem ser transformadas pelos materiais e pelos meios simbólicos que ele utiliza em seu processo de mediação junto ao estudante. Há evidências obtidas a partir de programas psico-educativos, voltados ao desenvolvimento cognitivo, de que a estrutura cognitiva dos aplicadores dos programas pode ser alterada, de modo que os efeitos de uma intervenção cognitiva não se situam apenas junto ao público-alvo imediato, os aplicandos (Feuerstein e cols., 1980). Ao contrário, ocorrem implicações cognitivas relevantes aos aplicadores. Quando as ações dos aplicadores estão imbricadas às ferramentas cognitivas utilizadas por eles mesmos, há uma tendência deles serem mobilizados e vivenciarem uma alteração cognitiva estrutural (Kozulin, 2000).

Muitas vezes, o que está em jogo para o aluno é a aprendizagem de um determinado conteúdo. Por outro lado, o que está em jogo para o professor é a competência que o habilita a atuar diretamente no fluxo de desenvolvimento de seu aluno, de modo a propiciar uma ampliação na capacidade de aprendizagem e, por conseqüência, uma melhor aprendizagem dos conteúdos escolares. Ambas as situações podem ser mobilizadas através de bons softwares concebidos a partir da terceira onda. $O$ que é defendido nesse texto não é novidade para muitos professores que desejam que a sua prática educativa seja um meio capaz de alterar a forma de pensar dos alunos. Muitos desses professores sabem que o maior desafio se encontra na dificuldade em fazer com que o aluno se aproprie das ferramentas de pensamento, dos meios simbólicos presentes nos conteúdos curriculares escolares. Essa distância, na medida em que é rompida, tem como elemento fundamental o papel do professor como mediador que intencionalmente provoca uma aproximação dos meios simbólicos e catalisa a construção de instrumentos psicológicos. No entanto, ao utilizar materiais ricos, tais como certos softwares educacionais, é possível que o professor transforme o seu aluno, assim como se transforme, gerando uma nova forma de ensinar e uma nova forma de provocar o seu aluno a aprender. E dentro desse processo, é provável que o professor também tenha suas estruturas cognitivas transformadas, de modo que 0 ato educativo inicialmente centrado do professor para $\circ$ aluno volte para o próprio professor, em uma relação de desenvolvimento para ambos.

Ao lançar a questão dos softwares educacionais como instrumentos psicológicos, este texto tratou um ponto considerado crucial. Softwares educacionais são meios simbólicos que, se bem modelados, podem se tornar instrumentos psicológicos, ou seja, ferramentas de pensamento geradoras de novos níveis cognitivos, tanto para os alunos como para os professores. Concomitantemente, softwares educacionais são meios simbólicos que trazem estruturas cognitivas sociais que podem ser favoráveis ou desfavoráveis à formação da prática docente na função de desenvolvimento do estudante.

Esse texto defendeu $o$ argumento de que a terceira grande onda trouxe novidades importantes e um caminho mais propício ao uso dos softwares educativos. Através dessa nova tendência tem se tornado possível avaliar os softwares educacionais como estruturas sociais de pensamento capazes de disponibilizar potenciais de formação e transformação humana. Essa é uma visão mais sofisticada do papel dos softwares educacionais e vai muito além do 
uso banal do computador como um meio de meramente atrair a atenção dos alunos ou fazer com que eles não saiam de aula, não percam o interesse etc. Tratar os softwares educacionais como instrumentos psicológicos implica em comprometer-se, não somente incentivar o desenvolvimento dos estudantes, mas também em construir maneiras de alterar deliberadamente esse caminho, ou seja, de modificar intencionalmente o fluxo de desenvolvimento cognitivo dos alunos.

Talvez uma limitação possível da terceira onda seja a viabilidade de tal proposta. Dúvidas podem surgir a respeito da capacidade da educação escolar em alterar diretamente as estruturas cognitivas dos estudantes e ampliar a sua capacidade de aprendizagem. Afinal, pode-se defender a idéia de que as estruturas cognitivas e a capacidade de aprendizagem são exclusivamente geradas e modificadas profundamente apenas na própria cultura e nas relações sociais em que 0 sujeito está inserido, e não através de intervenções pedagógicas feitas diretamente pela escola. No entanto, há evidências consideráveis de que é possível alcançar esse objetivo através de materiais e recursos didáticos bem elaborados e estratégias bem delimitadas no campo da mediação fornecida pelo professor (Adey, 1997, 1999, 2003; Adey \& Shayer, 1994, 1997; Kozulin, 1997, 2000; Kozulin \& Rand, 2000; Shayer \& Beasley, 1987). Por haver pesquisas que mostram a viabilidade dessa proposta, potencializa a elaboração de questões. De fato, ainda não se sabe muito bem como os softwares podem ou devem ser usados para que eles sejam ferramentas cognitivas para o aluno e para o professor no momento da sala de aula. Essa condição é mantida principalmente porque não se tem conhecimento, efetivamente, como os meios simbólicos se articulam com os instrumentos psicológicos. Não se sabe, por exemplo, se os instrumentos psicológicos são traduções diretas dos meios simbólicos sociais ou, ao contrário, se são construções internas específicas, em que os meios simbólicos têm um papel apenas indireto de provocação.

Para complementar a questão dessa temática não se conhece ainda quais são as variáveis que efetivamente intervém na dinâmica entre os meios simbólicos e os instrumentos psicológicos. Kozulin
(2000) aposta no fator mediacional humano como a variável mais importante de interferência. Entretanto, faltam pesquisas capazes de encontrar evidências sobre essa aposta. Outra questão importante, e que remete a um argumento debatido neste texto, envolve $\circ$ estudo da interação entre o professor e o software educacional. É muito provável que a maioria das interações entre os professores e os softwares não seja capaz de gerar acoplamentos no sentido defendido neste texto. Compreender as relações e as situações emergentes que possibilitam a interação imbricada é um empreendimento relevante dentro da temática aqui defendida. Em suma, todas essas questões levantadas, assim como outras possíveis apontam para uma agenda de pesquisa capaz de analisar e compreender, em termos teóricos e de evidências, o pressuposto de que os softwares educacionais podem ser instrumentos psicológicos, tanto para alunos como para professores.

\section{Referências}

Adey, P. (1997). It all depends on the context, doesn't it? Searching for general, educable dragons. Studies in Science Education, 29, 45-92.

Adey, P. (1999). The science of thinking, and science for thinking: a description of Cognitive Acceleration through Science Education (CASE). Geneva: UNESCO, International Bureau of Education.

Adey, P. (2003). Changing Minds. Disponível: http:// www.kcl.ac.uk/depsta/education/publications/ changing_minds.doc.pdf. Recuperado: 30 jul. 2003.

Adey, P., \& Shayer, M. (1994). Really raising standards: cognitive intervention and academic achievement. London: Routledge.

Adey, P., \& Shayer, M. (1997). Aceleración cognitiva por la enseñanza de las ciencias: CASE (Cognitive Acceleration in Science Education). Em: Es modificable la inteligencia? (pp. I57170). Madrid: Bruño.

Andaloro, G., Bellomonte, L., \& Sperandeo-Mineo, R. M. (1997). A computer-based learning environment in the field of Newtonian mechanics. International Journal of Science Education, 19, 66 I-680.

Beichner, R. (1994). Testing student interpretation of kinematics graphs. American Journal of Physics, 62, 750-762. 
Berger, C. F., Lu, C. R., Belzer, S. J., \& Voss, B. E. (1994). Research on the uses of technology in science education. Em D. L. Gabel (Ed.), Handbook on research on science teaching and learning (pp. 466-490). New York: Macmillan.

Bliss, J., \& Ogborn, J. ( 1992). Steps towards the formalisation of a psycho-logic of motion. Em A. Tiberghien \& H. Mandl (Orgs.), Intelligent learning environments and knowledge acquisition in physics (pp. 65-90). New York: Springer-Verlag.

Courtois, J. (1992). Practical work aid: knowledge representation in a model based AI System. Em A. Tiberghien \& H. Mandl, Intelligent learning environments and knowledge acquisition in physics (pp. 2 I-34). New York: Springer-Verlag.

Cotton, K. (199I). Computer-Assisted Instruction. School Improvement Research Series (SIRS). [On-line]. Disponível: http://wnww.nwrel.org/ scpd/sirs/5/cul0.html. Recuperado: 10 dez. 2007.

De Jong, T., \& Van Joolingen, W. R. (1998). Scientific discovery learning with computer simulations of conceptual domains. Review of Educational Research, 68, I79-202.

Doerr, H. M. (1997). Experiment, simulation and analysis: an integrated instructional approach to the concept of force. International Journal of Science Education, 19(3), 265-282.

Driver, R., \& Easley, J. (1978). Pupils and paradigms: a review of literature related to concept development in adolescent science students. Studies in Science Education, 5, 61-84.

Feuerstein, R., Rand, Y., Hoffman, M. B., \& Miller, R. (1980). Instrumental Enrichment: an intervention program for cognitive modifiability. Glenview (Illinois): Scott, Foresman and Company.

Feuerstein, R., Rand, Y., \& Rynders, J. E. (1988). Don't accept me as I am: helping 'retarded' people to excel. New York: Plenum.

Hartley, J. (2007). Teaching, learning and new technology: a review for teachers. British Journal of Educational Technology, 38(1), 42-62.

Hron, A. (1992). Simultaneos processing of different problem aspects in expert problem solving: an analysis in the domain of physics on the basis of formal theories of commonsense knowledge. Em A. Tiberghien \& H. Mandl (Orgs.), Intelligent learning environments and knowledge acquisition in physics (pp. 35-46). New York: Springer-Verlag.

Jonassen, D. H. (1992). What are cognitive tools? Em H. J. David (Ed.), Cognitive tools for learning (pp. I-6). New York: Springer-Verlag.

Jonassen, D. H. (1996). MindTools: computers in the classroom: MindTools for critical thinking. New Jersey: Englwood Cliffs.
Karlgren, K. (2006). E-learning acronyms. [On-line]. Disponível: http://people.dsv.su.se/ klas/Learn/index.html. Recuperado: 10 dez. 2007.

Kozma, R. B. (I99I). Learning with media. Review of Educational Research, 6I, I79-2II.

Kozulin, A. (Org.). (1997). The Ontogeny of Cognitive Modifiability: Applied Aspects of Mediated Learning Experience and Instrumental Enrichment. Jerusalem: ICELP.

Kozulin, A. (1999). Vygotsky's psychology: a biography of ideas. Cambridge: Harvard Univesity Press.

Kozulin, A. (2000). Instrumentos Psicológicos: la educación desde uma perspectiva sociocultural. Barcelona: Paidós.

Kozulin, A., \& Rand, Y. (Orgs.). (2000). Experience of Mediated Learning: An Impact of Feuerstein's Theory in Education and Psychology. Oxford: Pergamon Press.

Kubicek, J. P. (2005). Inquiry-based learning, the nature of science, and computer technology: new possibilities in science education. Canadian Journal of Learning and Technology, 3 I ( I), [On-line]. Disponível: http://www.cjlt.ca/content/vol3I.I/ kubicek.html. Recuperado: 10 dez. 2007.

Mandl, H., \& Hron, A. ( 1992). Cognitive theories as a basis for student modelling. Em A. Tiberghien \& H. Mandl (Orgs.), Intelligent learning environments and knowledge acquisition in physics (pp. I I - I22). New York: Springer-Verlag.

Mayes, J. T. (1992). Cognitive tools: a suitable case for learning. Em H. J. David (Ed.), Cognitive tools for learning (pp. 7-18). New York: Springer-Verlag.

Nirmalakhandan, N. (2007). Computerized adaptive tutorials to improve and assess problem-solving skills. Computers \& Education, 49(4), I32I-1329.

Roth, W. M. (1995). Affordances of computers in teacherstudent interactions: the case of Interactive physics. Journal of Research in Science Teaching, 32, 329-347.

Solomon, J. (1994). The rise and fall of constructivism. Studies in Science Education, 23, I - 19.

Salomon, G., Perkins, D. N., \& Globerson, T. (1991). Partners in cognition: extending human intelligence with intelligent technologies. Educational Researcher, 20(3), 2-9.

Schroeder, D. V., \& Moore, T. A. (1993). A computer-simulated Stern-Gerlach laboratory. American Journal of Physics, 6 I , 798805.

Schur, Y. (1997). Constructivism and mediated learning principles in science teaching. Em A. Kozulin (Ed.), The ontogeny 
of cognitive modifiability: applied aspects of Mediated Learning Experience and Instrumental Enrichment.(47-78) Jerusalém: ICELP.

Shayer, M., \& Beasley, F. (1987). Does Instrumental Enrichment work? British Educational Research Journal, 2(I3), I0 I- I 19.

Sjøberg, S. (2007). Constructivism and learning. Em E. Baker, B. McGaw \& P. Peterson (Orgs.), International Encyclopaedia of Education. Oxford: Elsevier. [On-line]. Disponível: http:// folk.uio.no/sveinsj/Constructivism_and_learning_Sjoberg.pdf. Recuperado: 10 dez. 2007.

Thornton, R. K., \& Sokoloff, D. R. (1990). Learning motion concepts using real-time microcomputer-based laboratory tools. American Journal of Physics, 58, 858-867.

Tisseau, G. (1992). Modelis: an artificial intelligence system which models thermodynamics textbook problems. Em A. Tiberghien \& H. Mandl (Orgs.), Intelligent learning environments and knowledge acquisition in physics (pp. 47-6I). New York: Springer-Verlag.

Vasconcelos, C., Praia, J. F., \& Almeida, L. S. (2003). Teorias de aprendizagem e o ensino/aprendizagem das ciências: da instrução à aprendizagem. Psicologia Escolar e Educacional, 7(I), II-19.

Vygotsky, L. S. (1978). Mind in Society: The Development of Higher Psychological Processes. Cambridge: Harvard University Press.

Vygotsky, L. S. (1986). Thought and Language. Cambridge: MIT Press.

Vygotsky, L. S. (1987). The Collected Works of L.S. Vygotsky: Problems of General Psychology. (vol. I). New York: Plenum Press.

Wertsch, J. V. (1985). Vygotsky and the social formation of mind. Cambridge: Harvard University Press.

Whithaus, C. (2004). The Development of Early ComputerAssisted Writing Instruction (1960-1978): The Double Logic of Media and Tools. Computers and the Humanities, 38, I49162.

Zinchenko, V. P. (1985). Vygotsky's ideas about units for the analysis of mind. Em J. V. Wertsch (Ed.), Culture, communication, and cognition: a vygotskian perspective (pp. 94-I I8). Cambridge: University Press.

Recebido em: 21/05/2007

Revisado em: 28/06/2007

Aprovado em: 15/0I/2008

Sobre o autor

Cristiano Mauro Assis Gomes (cristianogomes@ufmg.br) - Professor da Universidade Federal de Minas Gerais 